\title{
Diagnosis of Pure Ulnar Sensory Neuropathy Around the Hypothenar Area Using Orthodromic Inching Sensory Nerve Conduction Study: A Case Report
}

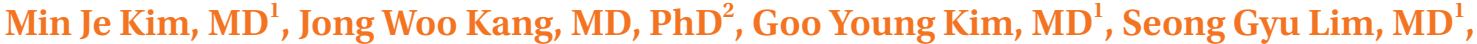 \\ Ki Hoon Kim, MD, PhD ${ }^{1}$, Byung Kyu Park, MD, $\mathrm{PhD}^{1}$, Dong Hwee Kim, MD, PhD ${ }^{1}$
}

Departments of ${ }^{1}$ Physical Medicine and Rehabilitation, ${ }^{2}$ Orthopedic Surgery, Korea University Ansan Hospital, Ansan, Korea

Ulnar neuropathy at the wrist is an uncommon disease and pure ulnar sensory neuropathy at the wrist is even rarer. It is difficult to diagnose pure ulnar sensory neuropathy at the wrist by conventional methods. We report a case of pure ulnar sensory neuropathy at the hypothenar area. The lesion was localized between $3 \mathrm{~cm}$ and $5 \mathrm{~cm}$ distal to pisiform using orthodromic inching test of ulnar sensory nerve to stimulate at three points around the hypothenar area. Ultrasonographic examination confirmed compression of superficial sensory branch of the ulnar nerve. Further, surgical exploration reconfirmed compression of the ulnar nerve. This case report demonstrates the utility of orthodromic ulnar sensory inching test.

Keywords Ulnar nerve compression syndromes, Wrist, Electrodiagnosis

\section{INTRODUCTION}

Ulnar neuropathy at the wrist (UNW) is not a common disease and pure ulnar sensory neuropathy at the wrist is even less common $[1,2]$. The ulnar nerve enters the Guyon's canal at the pisiform and divides into superficial and deep fibers in the pisohamate tunnel [1]. It is not easy to diagnose UNW based on conventional ulnar motor and sensory conduction studies. Several short segment incremental studies (SSIS) localize the lesion site in UNW to overcome the limitations of conventional studies [3-5]. Initial SSIS around the wrist was introduced by McIntosh et al. [4], which was stimulated at $1 \mathrm{~cm}$ interval from $3 \mathrm{~cm}$ proximal to the distal wrist crease (DWC) to $4 \mathrm{~cm}$ distal to the DWC with first dorsal interosseous (FDI) recording. Seror [5] performed SSIS to stimulate 1

Received July 19, 2017; Accepted September 8, 2017

Corresponding author: Dong Hwee Kim

Department of Physical Medicine and Rehabilitation, Korea University Ansan hospital, 123 Jeokgeum-ro, Danwon-gu, Ansan 15355, Korea. Tel: +8231-412-5330, Fax: +82-31-412-5344, E-mail: rmkdh@korea.ac.kr

ORCID: Min Je Kim (http://orcid.org/0000-0003-3794-2192); Jong Woo Kang (http://orcid.org/0000-0002-7979-4311); Goo Young Kim (http://orcid. org/0000-0001-7698-2045); Seong Gyu Lim (http://orcid.org/0000-0002-3681-6429); Ki Hoon Kim (http://orcid.org/0000-0002-7257-3858); Byung Kyu Park (http://orcid.org/0000-0001-8445-7099); Dong Hwee Kim (http://orcid.org/0000-0002-8116-0078).

(c) This is an open-access article distributed under the terms of the Creative Commons Attribution Non-Commercial License (http://creativecommons.org/ licenses/by-nc/4.0) which permits unrestricted noncommercial use, distribution, and reproduction in any medium, provided the original work is properly cited. Copyright $\odot 2018$ by Korean Academy of Rehabilitation Medicine 
$\mathrm{cm}$ proximal to the DWC and 5 (or 7) $\mathrm{cm}$ distal to DWC stimulation. In the SSIS introduced by Kim et al. [3], the ulnar nerve was stimulated at 3 points: $3 \mathrm{~cm}$ distal to the pisiform, just lateral to the pisiform, and $2 \mathrm{~cm}$ proximal to the pisiform. Ulnar motor SSIS at wrist were conducted via FDI recording and ulnar sensory SSIS at wrist, in the 5th finger recording. Most of them evaluated deep ulnar motor fibers. Only a single study evaluated the superficial ulnar sensory fibers around pisiform [3], but its utility was confined to lesion localization between pisiform and $2 \mathrm{~cm}$ proximal to pisiform or between pisiform and $3 \mathrm{~cm}$ distal to pisiform. In our case, superficial ulnar sensory lesion around the hypothenar area below $3 \mathrm{~cm}$ distal to pisiform was not evaluated. We report a case of diagnosis of pure ulnar sensory neuropathy around the hypothenar area using orthodromic inching sensory nerve conduction test, confirmed by ultrasonographic examination and surgical exploration.

\section{CASE REPORT}

A 40-year-old female had tingling sensation on the right 4th and 5th fingers 5 months ago with no history of trauma. The manual muscle testing result was normal in the right upper extremity including FDI, abductor pollicis brevis (APB), and abductor digiti minimi (ADM) muscles.
Hypoesthesia was detected on the right 5th, ulnar side of 4th fingers and distal 2/3 hypothenar area (Fig. 1A). The Tinel sign was positive at the point between the upper and middle 1/3 of right hypothenar area (Fig. 1A). Deep tendon reflex was normoactive and no Hoffman reflex was detected, bilaterally. Conventional median and ulnar motor and sensory nerve conduction studies (NCS) of both upper extremity nerves were performed [6] (Table 1). Right median motor and sensory responses and both ulnar motor responses with ADM and FDI recordings were normal (Table 1). Right antidromic ulnar sensory response with 5th digit recording showed low amplitude (9 $\mu \mathrm{V})$ compared with the left $(43 \mu \mathrm{V})$. Both dorsal ulnar cutaneous sensory responses were not significant (right, 30 $\mu \mathrm{V}$; left, $34 \mu \mathrm{V}$ ). Lesion localization was performed using a modified orthodromic ulnar sensory inching test, with wrist recording bilaterally [7]. The ulnar sensory nerve was stimulated at 3 points: $3 \mathrm{~cm}, 5 \mathrm{~cm}$, and $7 \mathrm{~cm}$ distal to the pisiform (Fig. 1A). An abnormal conduction delay (0.6 $\mathrm{ms}$ ) and definite partial conduction block (81.1\%) between $3 \mathrm{~cm}$ and $5 \mathrm{~cm}$ distal to the pisiform were observed (Fig. 1B). Needle electromyography yielded no abnormal action potentials in the right biceps brachii, pronator teres, flexor carpi ulnaris, ADM, FDI, and APB muscles. Electrodiagnosis revealed incomplete right superficial ulnar sensory neuropathy around hypothenar area, which
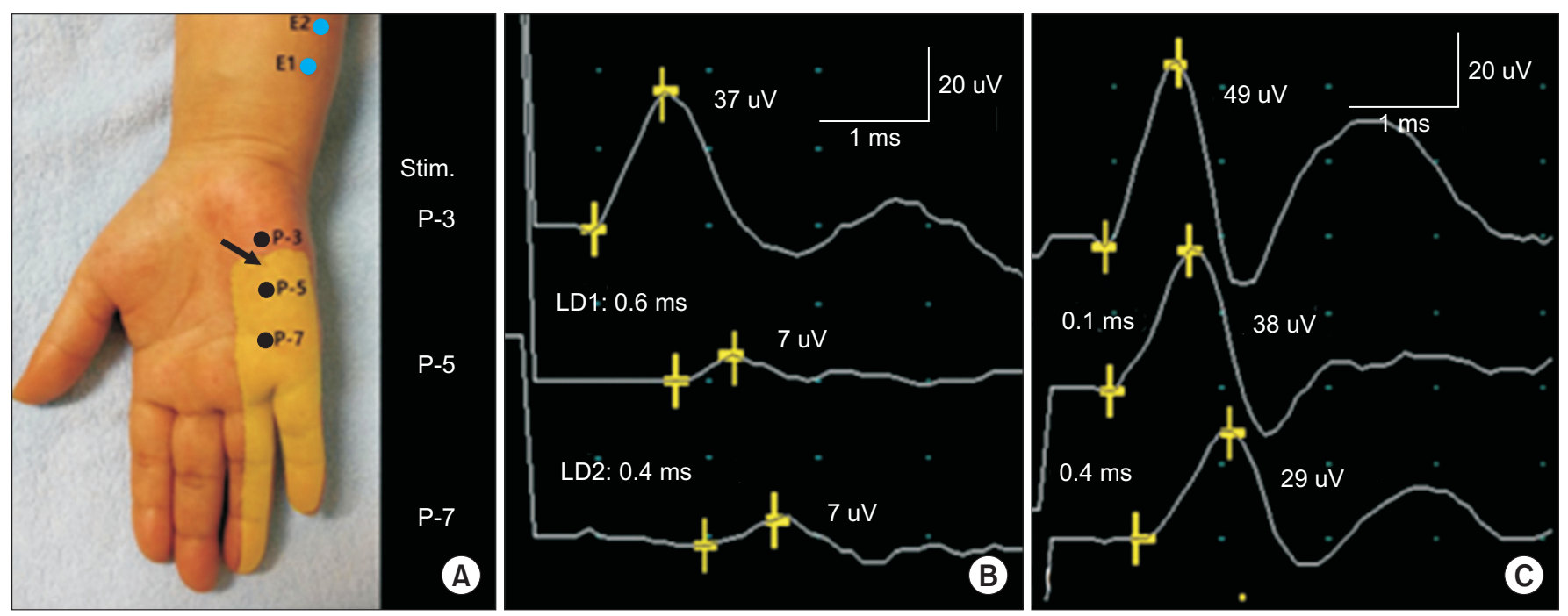

Fig. 1. (A) Orthodromic ulnar sensory inching test with distal forearm recording (E1 and E2) was conducted at three sites: $3 \mathrm{~cm}, 5 \mathrm{~cm}$, and $7 \mathrm{~cm}$ distal to pisiform (P-3, P-5 and P-7, respectively). Light yellow area represents hypoesthesia to touch and pinprick, and arrow indicates a positive Tinel sign. (B) Right orthodromic ulnar sensory inching test revealed abnormal conduction delay $(0.6 \mathrm{~ms})$ and definite partial conduction block $(81.1 \%)$ between $3 \mathrm{~cm}$ and $5 \mathrm{~cm}$ distal to pisiform compared with the left side (C). 
Table 1. Serial electrophysiological findings

\begin{tabular}{|c|c|c|c|c|c|c|c|c|c|}
\hline \multirow[b]{2}{*}{ Nerve tested } & \multirow[b]{2}{*}{$\begin{array}{l}\text { Stimula- } \\
\text { tion site }\end{array}$} & \multirow[b]{2}{*}{$\begin{array}{l}\text { Recording } \\
\text { site }\end{array}$} & \multicolumn{4}{|c|}{ Initial } & \multicolumn{3}{|c|}{ First FU } \\
\hline & & & $\begin{array}{l}\text { Lat }^{\text {a) }} \\
\text { (ms) }\end{array}$ & $\mathbf{A m p}^{\mathrm{b})}$ & $\begin{array}{c}\mathbf{C V} \\
(\mathbf{m} / \mathbf{s})\end{array}$ & $\begin{array}{l}\Delta \text { Lat } \\
(\mathbf{m s})\end{array}$ & $\begin{array}{l}\text { Lat }^{\text {a) }} \\
\text { (ms) }\end{array}$ & $\mathbf{A m p}^{\mathbf{b})}$ & $\begin{array}{c}\mathrm{CV} \\
(\mathrm{m} / \mathrm{s})\end{array}$ \\
\hline \multicolumn{10}{|l|}{ Motor } \\
\hline \multirow[t]{6}{*}{ Right ulnar } & Wrist & ADM & 2.9 & 10.0 & 59 & - & 2.8 & 9.0 & 59 \\
\hline & ME-3 & $\mathrm{ADM}$ & 6.1 & 9.6 & 61 & - & 6.0 & 8.5 & 61 \\
\hline & $\mathrm{ME}+7$ & $\mathrm{ADM}$ & 8.1 & 9.1 & - & - & 8.0 & 8.5 & - \\
\hline & Wrist & FDI & 3.5 & 11.0 & 58 & - & 3.4 & 9.2 & 58 \\
\hline & ME-3 & FDI & 6.9 & 9.2 & 53 & - & 6.7 & 8.4 & 52 \\
\hline & $\mathrm{ME}+7$ & FDI & 8.7 & 9.2 & - & - & 8.6 & 7.9 & - \\
\hline \multirow[t]{2}{*}{ Left ulnar } & Wrist & $\mathrm{ADM}$ & 2.8 & 12.1 & - & - & NT & NT & - \\
\hline & Wrist & FDI & 3.6 & 13.1 & - & - & NT & NT & - \\
\hline \multicolumn{10}{|l|}{ Sensory } \\
\hline Right ulnar & Wrist & 5 th digit & 3.1 & 9.0 & - & - & 3.3 & 9.0 & - \\
\hline Right DUCN & Wrist & 4th web space & 2.2 & 30.0 & - & - & NT & NT & - \\
\hline Left ulnar & Wrist & 5 th digit & 3.0 & 43.0 & - & - & NT & NT & - \\
\hline \multirow{3}{*}{$\begin{array}{l}\text { Right ulnar } \\
\text { orthodromic }\end{array}$} & P-3 & Wrist & 1.6 & 37 & - & 0.6 & NT & NT & - \\
\hline & P-5 & Wrist & 2.2 & 7 & - & 0.5 & NT & NT & - \\
\hline & P-7 & Wrist & 2.7 & 7 & - & - & NT & NT & - \\
\hline Left DUCN & Wrist & 4th web space & 2.0 & 34.0 & - & - & NT & NT & - \\
\hline \multirow{3}{*}{$\begin{array}{l}\text { Left ulnar } \\
\text { orthodromic }\end{array}$} & P-3 & Wrist & 1.6 & 49 & - & 0.1 & NT & NT & - \\
\hline & P-5 & Wrist & 1.7 & 38 & - & 0.4 & NT & NT & - \\
\hline & P-7 & Wrist & 2.1 & 29 & - & - & NT & NT & - \\
\hline
\end{tabular}

First FU, 4 weeks after initial study; Lat, latency; Amp, amplitude; CV, conduction velocity; ME-3, $3 \mathrm{~cm}$ distal to medial epicondyle; $\mathrm{ME}+7,7 \mathrm{~cm}$ proximal to medial epicondyle; ADM, abductor digiti minimi; FDI, first dorsal interosseous; DUCN, dorsal ulnar cutaneous nerve; P-3, $3 \mathrm{~cm}$ distal to pisiform; P-5, $5 \mathrm{~cm}$ distal to pisiform; P-7, $7 \mathrm{~cm}$ distal to pisiform; NT, not tested.

${ }^{a)}$ Onset latency in motor conduction studies and peak latency in sensory conduction studies.

${ }^{\mathrm{b}}$ Amplitudes are measured in millivolt ( $\mathrm{mV}$, motor) and in microvolt ( $\mu \mathrm{V}$, sensory).

was further confirmed by ultrasonography. The ultrasonographic examination (Accuvix V20, Medison, Seoul, Korea; 5-13 MHz linear probe) revealed compression of right superficial ulnar sensory branch $3.5 \mathrm{~cm}$ distal to pisiform and swelling of superficial ulnar sensory branch around upper 1/3 hypothenar area (Fig. 2A) with longitudinal and transverse view. Sonography-guided perineural injection with dexamethasone $2.5 \mathrm{mg}$ and $0.5 \%$ lidocaine $1 \mathrm{cc}$ around the compression site of the right ulnar sensory nerve ( $3.5 \mathrm{~cm}$ distal to pisiform), and oral medications including gabapentin and capsaicin cream for 1 month failed to ameliorate the symptoms. In a follow-up electrodiagnostic study after 4 weeks, there was no interval change compared with the initial study (Table 1). Sur- gical exploration around Guyon's canal and hypothenar area revealed a swelling of the superficial sensory branch of the ulnar nerve and fibrotic band-like structure compressing the superficial sensory branch (asterisk in Fig. 2B), which was compatible with the lesion site (around 3.5 $\mathrm{cm}$ distal to pisiform) detected by clinical (positive Tinel sign), electrophysiological (orthodromic inching sensory test), and ultrasonographic examinations. Further surgical exploration showed that the ulnar nerve was divided into deep motor and superficial branches below the hook of hamate. Surgical exploration and adhesiolysis alleviated the sensory symptoms. 

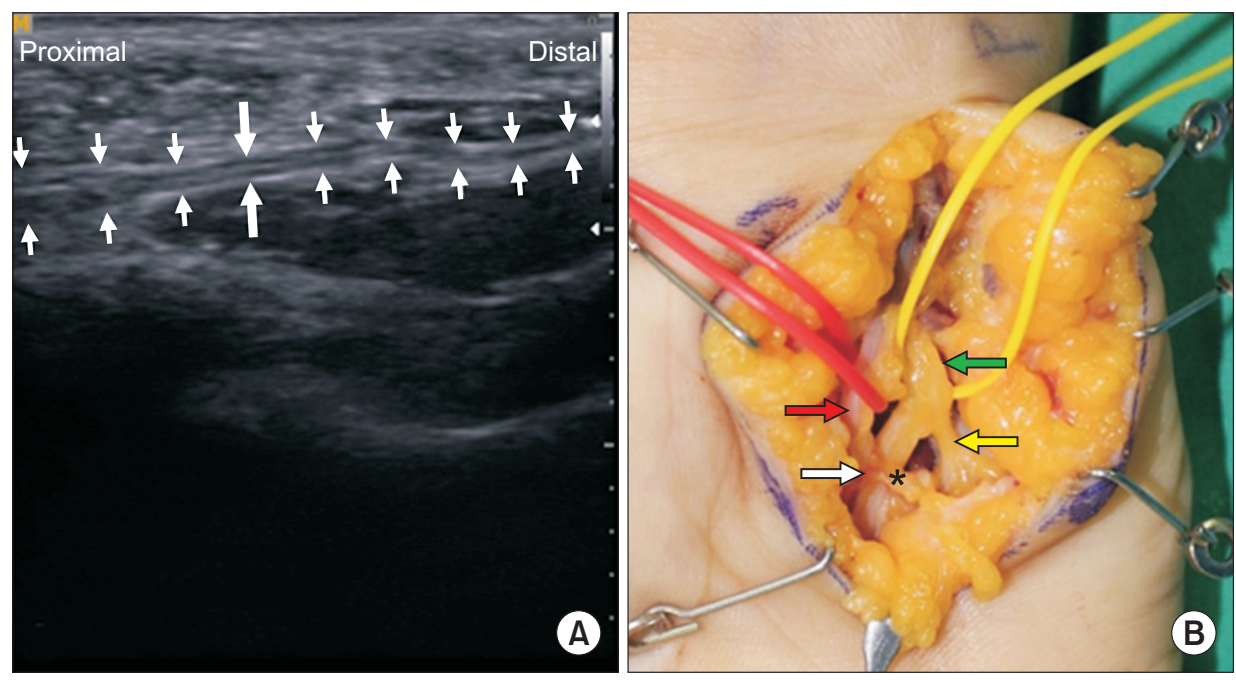

Fig. 2. (A) Ultrasonographic evaluation showed compression of right superficial ulnar sensory branch $3.5 \mathrm{~cm}$ distal to pisiform (long and thick arrows) and swelling of superficial ulnar sensory branch around upper 1/3 hypothenar area (thin arrows on the proximal side). (B) Surgical exploration demonstrated swelling of the superficial sensory branch of the ulnar nerve (white arrow) and fibrotic band-like structure compressing the superficial sensory branch (asterisk). Green arrow indicates the main trunk of ulnar nerve; yellow arrow, deep branch of ulnar nerve; and red arrow, ulnar artery.

\section{DISCUSSION}

In UNW, the least common form involves only the superficial sensory branch of the ulnar nerve [8]. Patients complain of tingling or sensory changes on the palmar side of hypothenar area and the 5th and 4th digits. Physical examination indicates sensory loss in the above area with preservation of intrinsic and extrinsic muscles of hand innervated by the ulnar nerve. In our case, clinical findings suggested a sensory form of UNW. A detailed physical examination demonstrated a distal $2 / 3$ hypothenar area and the ulnar side of 4 th and the 5th digits, and positive Tinel sign at the point between the upper $1 / 3$ and middle 1/3 hypothenar area on the right side (Fig. 1A).

Electrophysiological studies using ulnar motor nerve conduction with ADM and FDI recordings, and ulnar sensory and dorsal ulnar cutaneous sensory NCS are recommended for the investigation of UNW, which failed to localize the lesion site around the wrist [9]. In our case, conventional conduction studies of the ulnar nerve including median and ulnar NCS based on ADM and FDI recordings, revealed normal response of dorsal ulnar cutaneous sensory nerve except for low amplitude of the right ulnar sensory response. These electrophysiological findings suggested incomplete right ulnar sensory neuropathy; however, the lesion location was undetermined.
Based on the clinical findings including Tinel sign around the hypothenar area, an orthodromic inching test of ulnar sensory nerve with distal forearm recording for translesional study was designed (Fig. 1A). The new technique demonstrated definite partial conduction block between $3 \mathrm{~cm}$ and $5 \mathrm{~cm}$ distal to pisiform. Morphological changes in the ulnar nerve at the lesion site were demonstrated via ultrasonographic examination. To the best of our knowledge, this case represents pure ulnar sensory neuropathy around wrist. In fact, accurate localization of the lesion of superficial ulnar sensory neuropathy around the wrist is not easy using conventional NCS. The antidromic SSIS for superficial sensory branch of the ulnar nerve, which was previously developed was useful for the lesion around pisiform, that is, between pisiform and $2 \mathrm{~cm}$ proximal to pisiform and between pisiform and $3 \mathrm{~cm}$ distal to pisiform [3]. However, in patients with ulnar sensory nerve lesion around hypothenar area, the antidromic sensory SSIS was not useful because it evaluated only the lesion around Guyon's canal, that is, between $3 \mathrm{~cm}$ distal to pisiform and $2 \mathrm{~cm}$ proximal to pisiform. Furthermore, it was difficult to perform in the hypothenar lesion due to the short distance between the recording electrode and the stimulation sites. Orthodromic sensory technique was not preferred in conventional sensory NCS because of smaller amplitude compared with antidromic sensory 
technique. However, orthodromic ulnar sensory inching with stimulation of the hypothenar area and with distal forearm recording can be used in patients with suspected ulnar sensory neuropathy around hypothenar area, and is especially useful in patients with conduction block or delay around hypothenar area. We hope to conduct the orthodromic ulnar sensory inching test around hypothenar area in healthy subjects for normative reference values, in a future study.

Ultrasonographic examination is a powerful tool to evaluate the morphological changes of the superficial sensory nerves or their surrounding soft tissues [10]. In our patient, ultrasonographic examination demonstrated compression of superficial sensory branch of the ulnar nerve around the lesion site and its proximal swelling using the orthodromic ulnar sensory inching test, which was confirmed with surgical exploration and adhesiolysis (Fig. 2). Further surgical exploration showed ulnar nerve compression by the ulnar artery branch.

Surgical exploration demonstrated swelling of the superficial sensory branch of the ulnar nerve (white arrow) and fibrotic band-like structure compressing the superficial sensory branch (asterisk). The green arrow indicates the main trunk of the ulnar nerve; the yellow arrow, the deep branch of ulnar nerve; and the red arrow, ulnar artery (Fig. 2).

In summary, our case represents a very uncommon incidence of pure ulnar sensory neuropathy involving the hypothenar area, which was the first case established by ultrasonographic examination and surgical exploration. Orthodromic inching test of ulnar nerve distal to pisiform is a very useful technique in patients with typical symptoms of ulnar neuropathy without deficits of ADM and FDI or significant findings in routine NCS and needle electromyography and the previous developed SSIS for ulnar sensory nerve around wrist [3]. Furthermore, orthodromic inching test supplemented by ultrasonographic examination plays a very important role in determining treatment options.

\section{CONFLICT OF INTEREST}

No potential conflict of interest relevant to this article was reported

\section{REFERENCES}

1. Preston DC, Shapiro BE. Electromyography and neuromuscular disorders: clinical-electrophysiologic correlations. 3rd ed. London: Elsevier; 2013. p. 319.

2. Olney RK, Hanson M. AAEE case report \#15: ulnar neuropathy at or distal to the wrist. Muscle Nerve 1988; 11:828-32.

3. Kim DH, Kang YK, Hwang M, Kwon HK, Lee HJ, Kim BG. Reference values of fractionated neurography of the ulnar nerve at the wrist in healthy subjects. Clin Neurophysiol 2005;116:2853-7.

4. McIntosh KA, Preston DC, Logigian EL. Short-segment incremental studies to localize ulnar nerve entrapment at the wrist. Neurology 1998;50:303-6.

5. Seror P. Electrophysiological pattern of 53 cases of ulnar nerve lesion at the wrist. Neurophysiol Clin 2013; 43:95-103.

6. Lee HJ, DeLisa JA. Manual of nerve conduction study and surface anatomy for needle electromyography. 4th ed. Philadelphia: Lippincott Williams \& Wilkins; 2005. p. 25-27, 34, 36-38, 45-49, 52-55.

7. Kim Y, Kim DH, Kim NH, Kim BH, Park BK. Dorsal sural neuropathy: electrophysiological, ultrasonographic, and magnetic resonance imaging findings. Muscle Nerve 2012;46:597-600.

8. Dumitru D, Amato AA, Zwarts MJ. Electrodiagnostic medicine. 2nd ed. Philadelphia: Hanley \& Belfus; 2002. p. 1084.

9. Preston DC, Shapiro BE. Electromyography and neuromuscular disorders: clinical-electrophysiologic correlations. 3rd ed. London: Elsevier; 2013. p. 322-23.

10. Kim KH, Park BK, Kim DH. A case of lateral calcaneal neuropathy: lateral heel pain. Muscle Nerve 2016;54: 801-4. 